EUROPEAN JOURNAL OF PURE AND APPLIED MATHEMATICS

Vol. 11, No. 1, 2018, 189-201

ISSN 1307-5543 - www.ejpam.com

Published by New York Business Global

\title{
Convergence Rate of Implicit Iteration Process and a Data Dependence Result
}

\author{
Isa Yildirim ${ }^{1, *}$, Mujahid Abbas ${ }^{2}$ \\ 1 Department of Mathematics, Faculty of Science, Ataturk University, Erzurum 25240, \\ Turkey \\ ${ }^{2}$ Department of Mathematics, GC University, Katchery Road, Lahore 54000, Pakistan
}

\begin{abstract}
The aim of this paper is to introduce an implicit S-iteration process and study its convergence in the framework of W-hyperbolic spaces. We show that the implicit S-iteration process has higher rate of convergence than implicit Mann type iteration and implicit Ishikawatype iteration processes. We present a numerical example to support the analytic result proved herein. Finally, we prove a data dependence result for a contractive type mapping using implicit S-iteration process.
\end{abstract}

2010 Mathematics Subject Classifications: AMS 47H10, 54H25

Key Words and Phrases: Implicit iterations, convergence rate, data dependence

\section{Introduction and preliminaries}

Throughout this paper, the letter $\mathbb{N}$ will denote the set of natural numbers.

The theory of fixed points deals with the conditions which guarantee that a mapping $T$ of a set $X$ into itself admits one or more fixed points, that is, points $x$ of $X$ which solve an operator equation $x=T x$, called a fixed point equation. Fixed point theory serves as an essential tool for solving problems arising in various branches of mathematical analysis. Over the past two decades the development of fixed point theory in metric spaces has attracted considerable attention due to numerous applications in areas such as variational and linear inequalities, optimization, and approximation theory.

The set $\{p \in X: p=T p\}$ of all fixed points of $T$ is denoted by $F(T)$.

One of the basic and the most widely applied fixed point theorem in all of analysis is "Banach ( or Banach- Cassioppoli ) Contraction principle" due to Banach [2]. This principle lies at the heart of metric fixed point theory. It states that if $(X, d)$ is a complete metric space and $T: X \rightarrow X$ satisfies

$$
d(T x, T y) \leq k d(x, y)
$$

${ }^{*}$ Corresponding author.

Email addresses: isayildirim@atauni.edu.tr (I. Yildirim), abbas.mujahid@gmail.com (M. Abbas) 
for all $x, y \in X$, with $k \in(0,1)$, then $T$ has a unique fixed point. Due to its applications in mathematics and other related disciplines, Banach contraction principle has been generalized in many directions.

Zamfirescu [21] obtained an important generalization of Banach fixed point theorem using Zamfirescu mapping.

A self mapping $T$ on a metric space $X$ is called Zamfirescu mapping if there exist real numbers $a, b, c$ satisfying $0<a<1,0<b, c<1 / 2$ such that at least one of the following is true:

$$
\left\{\begin{array}{cc}
\left(z_{1}\right) & d(T x, T y) \leq a d(x, y) \\
\left(z_{2}\right) & d(T x, T y) \leq b(d(x, T x)+d(y, T y)) \\
\left(z_{3}\right) & d(T x, T y) \leq c(d(x, T y)+d(y, T x)) .
\end{array}\right.
$$

for any $x, y \in X$.

The contractive condition (1) can be reformulated as follows:

$$
\left\{\begin{array}{c}
\left(b_{1}\right) \quad d(T x, T y) \leq \delta d(x, y)+2 \delta d(x, T x) \text { if one uses }\left(z_{2}\right), \text { and } \\
\left(b_{2}\right) \quad d(T x, T y) \leq \delta d(x, y)+2 \delta d(x, T y) \text { if one uses }\left(z_{3}\right)
\end{array}\right.
$$

for all $x, y \in X$, where $\delta=\max \left\{a, \frac{b}{1-b}, \frac{c}{1-c}\right\}([4])$. Clearly, $\delta \in[0,1)$.

A mapping satisfying condition $\left(b_{1}\right)$ or $\left(b_{2}\right)$ is called a quasi-contractive mapping. This class of mappings is general than the class of Zamfirescu mappings.

Osilike and Udomene [17] extended the above class of mappings and introduced a mapping $T$ satisfying the following contractive condition:

$$
d(T x, T y) \leq \delta d(x, y)+L d(x, T x),
$$

for all $x, y \in X$, where $L \geq 0$ and $\delta \in[0,1)$.

For more results and discussion in this direction, we refer to [1], [13] and references mentioned therein.

Imoru and Olantiwo [10] gave the following definition:

Definition 1. A self mapping $T$ on $X$ is called a contractive-like mapping if there exists a constant $\delta \in[0,1)$ and a strictly increasing and continuous function $\varphi:[0, \infty) \rightarrow[0, \infty)$ with $\varphi(0)=0$ such that for any $x, y \in X$, we have

$$
d(T x, T y) \leq \delta d(x, y)+\varphi(d(x, T x)) .
$$

Definition 2. [18] Let $T, S$ be two self mappings on $X$. We say that $S$ is an approximate operator of $T$ if for all $\varepsilon>0$, we have $d(T x, S x) \leq \varepsilon$ holds for any $x \in X$.

An ambient space equipped with certain convexity structure play a significant role in solving a fixed point equation. Since Banach space is a vector space, the concept of a line segment joining any two points of a nonempty subset of a Banach space give rise to the convexity structure. However, metric spaces do not naturally have this convex structure. The notion of convex metric spaces was introduced by Takahashi [19] who studied the fixed points of nonexpansive mappings in the setting of such spaces. All normed spaces 
and their convex subsets are convex metric spaces. But there are many examples of convex metric spaces which are not embedded in any normed space ([19]). Over time, different convex structures have been introduced on metric spaces.

Kohlenbach [15] introduced $W$-hyperbolic spaces as follows:

Definition 3. $A W$-hyperbolic space $(X, d, W)$ is a metric space $(X, d)$ together with a convexity mopping $W: X^{2} \times[0,1] \rightarrow X$ satisfying the following properties:

$$
\begin{aligned}
& \text { (i) } d(u, W(x, y, \alpha)) \leq(1-\alpha) d(u, x)+\alpha d(u, y) \\
& \text { (ii) } d(W(x, y, \alpha), W(x, y, \beta))=|\alpha-\beta| d(x, y) \\
& \text { (iii) } W(x, y, \alpha)=W(y, x, 1-\alpha) \\
& \text { (iv) } d(W(x, z, \alpha), W(y, w, \alpha)) \leq(1-\alpha) d(x, y)+\alpha d(z, w)
\end{aligned}
$$

for all $x, y, z, w \in X$ and $\alpha, \beta \in[0,1]$.

If the triplet $(X, d, W)$ satisfies the condition (i) only, then it coincides with the convex metric space introduced by Takahashi [19]. Every hyperbolic space is a convex metric space but converse does not hold in general ( $[6])$. A subset $E$ of a $W$-hyperbolic space $X$ is convex if $W(x, y, \alpha) \in E$ for all $x, y \in E$ and $\alpha \in[0,1]$.

Note that every $W$-hyperbolic space is a geodesic space. CAT(0) spaces, normed linear space, The Hilbert ball and Busseman spaces are important examples of $W$ - hyperbolic spaces. A $W$ - hyperbolic space represents a unified approach for both linear and nonlinear structures simultaneously. There are hyperbolic spaces which are not imbedded in any Banach space .

On the other hand, different iterative algorithms have been used to approximate the solution of a fixed point equation $([5,11,14,16,19])$. Implicit iterative schemes are of great importance from numerical stand point as they provide accurate approximation ( see, $[6],[12],[7,8,20])$.

The motivation of this paper is to define an implicit S-iteration process with higher rate of convergence when compared with Mann type(7) and Ishikawa type (6) implicit iterative processes.

Let $E$ be a nonempty convex subset of a $W$-hyperbolic space $X$ and $T: E \rightarrow E$. Choose $x_{0} \in E$ and define the sequence $\left\{x_{n}\right\}$ as follows:

$$
\begin{aligned}
& x_{n}=W\left(T x_{n-1}, T y_{n}, \alpha_{n}\right) \\
& y_{n}=W\left(x_{n}, T x_{n}, \beta_{n}\right), \quad n \in \mathbb{N},
\end{aligned}
$$

where $\left\{a_{n}\right\}$ and $\left\{\beta_{n}\right\}$ are certain real sequences in $[0,1]$.

We translate implicit Ishikawa and implicit Mann iteration processes introduced by Ćirić et al. $([8,9])$ in the setup of $W$-hyperbolic space as follows:

$$
\begin{aligned}
& x_{n}=W\left(x_{n-1}, T y_{n}, \alpha_{n}\right) \\
& y_{n}=W\left(x_{n}, T x_{n}, \beta_{n}\right) \quad n \in \mathbb{N},
\end{aligned}
$$

and

$$
x_{n}=W\left(x_{n-1}, T y_{n}, \alpha_{n}\right), n \in \mathbb{N} .
$$


Remark 1. Note that, the process (5) is independent of (6) and (7) in the sense that neither of them reduce to the other.

The following definition is due to Berinde [3].

Definition 4. Let $\left\{x_{n}\right\}$ and $\left\{u_{n}\right\}$ be two fixed point iteration processes in a metric space $X$ such that $\lim _{n \rightarrow \infty} x_{n}=\lim _{n \rightarrow \infty} u_{n}=p$, where $p$ is a fixed point of a self mapping $T$ on $X$. Suppose that

$$
d\left(x_{n}, p\right) \leq a_{n} \text { and } d\left(u_{n}, p\right) \leq b_{n}, n \in \mathbb{N} .
$$

where $\left\{a_{n}\right\}$ and $\left\{b_{n}\right\}$ are two null sequences of positive numbers. If $\left\{a_{n}\right\}$ converges faster than $\left\{b_{n}\right\}$, then we say $\left\{x_{n}\right\}$ converges faster than $\left\{u_{n}\right\}$ to $p$.

We also need the following lemma in order to prove our main results.

Lemma 1. [18] Let $\left\{a_{n}\right\}$ be a nonnegative sequence. If there exists an $n_{0} \in \mathbb{N}$ such that for all $n \geq n_{0}$, we have

$$
a_{n+1} \leq\left(1-\mu_{n}\right) a_{n}+\mu_{n} \eta_{n},
$$

where $\mu_{n} \in(0,1), \sum_{n=0}^{\infty} \mu_{n}=\infty$ and $\eta_{n} \geq 0$ for all $n \in \mathbb{N}$. Then the following holds:

$$
0 \leq \lim _{n \rightarrow \infty} \sup a_{n} \leq \lim _{n \rightarrow \infty} \sup \eta_{n} .
$$

\section{Main Results}

We start with the following result.

Theorem 1. Let $E$ be a nonempty closed convex subset of $W$-hyperbolic space $X$ and $T: E \rightarrow E$ a contractive type mapping with $F(T) \neq \emptyset$. Then, for the sequence $\left\{x_{n}\right\}$ defined in (5) with $\sum\left(1-\alpha_{n}\right)=\infty$, we have $\lim _{n \rightarrow \infty} x_{n}=p$, where $p \in F(T)$.

Proof. Suppose that $p \in F(T)$. Using (5) and (4), we have

$$
\begin{aligned}
d\left(x_{n}, p\right) \leq & d\left(W\left(T x_{n-1}, T y_{n}, \alpha_{n}\right), p\right) \\
\leq & \alpha_{n} d\left(T x_{n-1}, p\right)+\left(1-\alpha_{n}\right) d\left(T y_{n}, p\right) \\
\leq & \alpha_{n}\left[\delta d\left(x_{n-1}, p\right)+\varphi(d(p, T p))\right] \\
& +\left(1-\alpha_{n}\right)\left[\delta d\left(y_{n}, p\right)+\varphi(d(p, T p))\right] \\
= & \alpha_{n} \delta d\left(x_{n-1}, p\right)+\left(1-\alpha_{n}\right) \delta d\left(y_{n}, p\right)
\end{aligned}
$$

and

$$
\begin{aligned}
d\left(y_{n}, p\right) & =d\left(W\left(x_{n}, T x_{n}, \beta_{n}\right), p\right) \\
& \leq \beta_{n} d\left(x_{n}, p\right)+\left(1-\beta_{n}\right) d\left(T x_{n}, p\right) \\
& \leq \beta_{n} d\left(x_{n}, p\right)+\left(1-\beta_{n}\right)\left[\delta d\left(x_{n}, p\right)+\varphi(d(p, T p))\right] \\
& =\beta_{n} d\left(x_{n}, p\right)+\left(1-\beta_{n}\right) \delta d\left(x_{n}, p\right) \\
& =\left[\beta_{n}+\left(1-\beta_{n}\right) \delta\right] d\left(x_{n}, p\right) .
\end{aligned}
$$


Therefore,

$$
d\left(x_{n}, p\right) \leq \alpha_{n} \delta d\left(x_{n-1}, p\right)+\left(1-\alpha_{n}\right) \delta\left[\beta_{n}+\left(1-\beta_{n}\right) \delta\right] d\left(x_{n}, p\right) .
$$

That is,

$$
\left[1-\left(1-\alpha_{n}\right) \delta\left[\beta_{n}+\left(1-\beta_{n}\right) \delta\right]\right] d\left(x_{n}, p\right) \leq \alpha_{n} \delta d\left(x_{n-1}, p\right),
$$

which further implies that

$$
d\left(x_{n}, p\right) \leq \frac{\alpha_{n} \delta}{1-\left(1-\alpha_{n}\right) \delta\left[\beta_{n}+\left(1-\beta_{n}\right) \delta\right]} d\left(x_{n-1}, p\right) .
$$

We set

$$
\Delta_{n}=\frac{\alpha_{n} \delta}{1-\left(1-\alpha_{n}\right) \delta\left[\beta_{n}+\left(1-\beta_{n}\right) \delta\right]}
$$

Then

$$
\begin{aligned}
1-\Delta_{n} & =1-\frac{\alpha_{n} \delta}{1-\left(1-\alpha_{n}\right) \delta\left[\beta_{n}+\left(1-\beta_{n}\right) \delta\right]} \\
& =\frac{1-\left(1-\alpha_{n}\right) \delta\left[\beta_{n}+\left(1-\beta_{n}\right) \delta\right]-\alpha_{n} \delta}{1-\left(1-\alpha_{n}\right) \delta\left[\beta_{n}+\left(1-\beta_{n}\right) \delta\right]} \\
& \geq 1-\left(1-\alpha_{n}\right) \delta\left[\beta_{n}+\left(1-\beta_{n}\right) \delta\right]-\alpha_{n} \delta
\end{aligned}
$$

implies that

$$
\begin{aligned}
\Delta_{n} & \leq\left(1-\alpha_{n}\right) \delta\left[\beta_{n}+\left(1-\beta_{n}\right) \delta\right]+\alpha_{n} \delta \\
& =\left(1-\alpha_{n}\right) \delta\left[1-(1-\delta)\left(1-\beta_{n}\right)\right]+\alpha_{n} \delta \\
& \leq\left(1-\alpha_{n}\right) \delta+\alpha_{n} \\
& =1-\left(1-\alpha_{n}\right)(1-\delta) .
\end{aligned}
$$

By (11) and (12), we have

$$
\begin{aligned}
d\left(x_{n}, p\right) & \leq\left[1-\left(1-\alpha_{n}\right)(1-\delta)\right] d\left(x_{n-1}, p\right) \\
& \leq \prod_{i=1}^{n}\left[1-\left(1-\alpha_{i}\right)(1-\delta)\right] d\left(x_{0}, p\right) .
\end{aligned}
$$

Since $a>0,1+a \leq e^{a}$, (13) gives that

$$
\begin{aligned}
d\left(x_{n}, p\right) & \leq \exp \left\{\sum_{i=1}^{n}\left(1-\alpha_{i}\right)(1-\delta)\right\} d\left(x_{0}, p\right) \\
& \leq \exp \left\{\sum_{n=1}^{\infty}\left(1-\alpha_{n}\right)(1-\delta)\right\} d\left(x_{0}, p\right) .
\end{aligned}
$$

Using the fact that $0 \leq \delta<1$ and $\sum\left(1-\alpha_{n}\right)=\infty$, we conclude that $\lim _{n \rightarrow \infty} d\left(x_{n}, p\right)=0$. 
The following result deals with the rate of convergence of implicit S-iteration process.

Theorem 2. Let $E$ be a nonempty closed convex subset of $W$-hyperbolic space $X$ and $T: E \rightarrow E$ a contractive type mapping with $F(T) \neq \emptyset$. Then, the sequence $\left\{x_{n}\right\}$ defined in (5) with $\sum\left(1-\alpha_{n}\right)=\infty$ converges to the fixed point of $T$ faster than implicit Ishikawa type (6) and implicit Mann type (7) iterations.

Proof. Let $p$ be a fixed point of T. Using an implicit Ishikawa type (6) iteration process we have,

$$
\begin{aligned}
d\left(x_{n}, p\right) & =d\left(W\left(x_{n-1}, T y_{n}, \alpha_{n}\right), p\right) \\
\leq & \alpha_{n} d\left(x_{n-1}, p\right)+\left(1-\alpha_{n}\right) d\left(T y_{n}, p\right) \\
\leq & \alpha_{n} d\left(x_{n-1}, p\right)+\left(1-\alpha_{n}\right)\left[\delta d\left(y_{n}, p\right)+\varphi(d(p, T p))\right] \\
& =\alpha_{n} d\left(x_{n-1}, p\right)+\left(1-\alpha_{n}\right) \delta d\left(y_{n}, p\right)
\end{aligned}
$$

and

$$
\begin{aligned}
d\left(y_{n}, p\right) & =d\left(W\left(x_{n}, T x_{n}, \beta_{n}\right), p\right) \\
& \leq \beta_{n} d\left(x_{n}, p\right)+\left(1-\beta_{n}\right) d\left(T x_{n}, p\right) \\
& \leq \beta_{n} d\left(x_{n}, p\right)+\left(1-\beta_{n}\right)\left[\delta d\left(x_{n}, p\right)+\varphi(d(p, T p))\right] \\
& =\beta_{n} d\left(x_{n}, p\right)+\left(1-\beta_{n}\right) \delta d\left(x_{n}, p\right) \\
& =\left[\beta_{n}+\delta\left(1-\beta_{n}\right)\right] d\left(x_{n}, p\right) .
\end{aligned}
$$

Therefore,

$$
d\left(x_{n}, p\right) \leq \alpha_{n} d\left(x_{n-1}, p\right)+\left(1-\alpha_{n}\right) \delta\left[\beta_{n}+\delta\left(1-\beta_{n}\right)\right] d\left(x_{n}, p\right) .
$$

That is,

$$
\begin{aligned}
d\left(x_{n}, p\right) & \leq \frac{\alpha_{n}}{1-\left(1-\alpha_{n}\right) \delta\left[\beta_{n}+\delta\left(1-\beta_{n}\right)\right]} d\left(x_{n-1}, p\right) \\
& \leq \ldots \leq c_{n}
\end{aligned}
$$

where

$$
c_{n}=\left(\frac{\alpha_{n}}{1-\left(1-\alpha_{n}\right) \delta\left[\beta_{n}+\delta\left(1-\beta_{n}\right)\right]}\right)^{n} d\left(x_{0}, p\right) .
$$

Using implicit Mann iteration (6), we obtain that

$$
\begin{aligned}
d\left(x_{n}, p\right) & =d\left(W\left(x_{n-1}, T x_{n}, \alpha_{n}\right), p\right) \\
& \leq \alpha_{n} d\left(x_{n-1}, p\right)+\left(1-\alpha_{n}\right) d\left(T x_{n}, p\right) \\
& \leq \alpha_{n} d\left(x_{n-1}, p\right)+\left(1-\alpha_{n}\right)\left[\delta d\left(x_{n}, p\right)+\varphi(d(p, T p))\right] \\
& =\alpha_{n} d\left(x_{n-1}, p\right)+\left(1-\alpha_{n}\right) \delta d\left(x_{n}, p\right) .
\end{aligned}
$$

Thus, we have

$$
d\left(x_{n}, p\right) \leq \frac{\alpha_{n}}{1-\left(1-\alpha_{n}\right) \delta} d\left(x_{n-1}, p\right)
$$


I. Yildirim, M. Abbas / Eur. J. Pure Appl. Math, 11 (1) (2018), 189-201

$$
\leq b_{n}
$$

where

$$
b_{n}=\left(\frac{\alpha_{n}}{1-\left(1-\alpha_{n}\right) \delta}\right)^{n} d\left(x_{0}, p\right) .
$$

Using implicit S-iteration process and following the arguments of the proof of Theorem 1, we have

$$
\begin{aligned}
d\left(x_{n}, p\right) & \leq \frac{\alpha_{n} \delta}{1-\left(1-\alpha_{n}\right) \delta\left[\beta_{n}+\left(1-\beta_{n}\right) \delta\right]\left[\gamma_{n}+\left(1-\gamma_{n}\right) \delta\right]} d\left(x_{n-1}, p\right) \\
& \leq \ldots \leq a_{n}
\end{aligned}
$$

where

$$
a_{n}=\left(\frac{\alpha_{n} \delta}{1-\left(1-\alpha_{n}\right) \delta\left[\beta_{n}+\left(1-\beta_{n}\right) \delta\right]\left[\gamma_{n}+\left(1-\gamma_{n}\right) \delta\right]}\right)^{n} d\left(x_{0}, p\right) .
$$

Note that $\lim _{n \rightarrow \infty} \frac{a_{n}}{c_{n}}=0$ and $\lim _{n \rightarrow \infty} \frac{a_{n}}{b_{n}}=0$. Indeed,

$$
\begin{aligned}
{\left[\gamma_{n}+\left(1-\gamma_{n}\right) \delta\right] } & <1 \Rightarrow\left[\beta_{n}+\left(1-\beta_{n}\right) \delta\right]<1 \\
& \Rightarrow\left(1-\alpha_{n}\right) \delta\left[\beta_{n}+\left(1-\beta_{n}\right) \delta\right]<1 \\
& \Rightarrow 1-\left(1-\alpha_{n}\right) \delta\left[\beta_{n}+\left(1-\beta_{n}\right) \delta\right]>0
\end{aligned}
$$

give

$$
\begin{aligned}
\alpha_{n} \delta & <\alpha_{n} \Rightarrow \frac{\alpha_{n} \delta}{1-\left(1-\alpha_{n}\right) \delta\left[\beta_{n}+\delta\left(1-\beta_{n}\right)\right]} \\
& <\frac{\alpha_{n}}{1-\left(1-\alpha_{n}\right) \delta} \\
& \Rightarrow\left(\frac{\alpha_{n} \delta}{1-\left(1-\alpha_{n}\right) \delta\left[\beta_{n}+\delta\left(1-\beta_{n}\right)\right]}\right)^{n} \\
& <\left(\frac{\alpha_{n}}{1-\left(1-\alpha_{n}\right) \delta}\right)^{n}
\end{aligned}
$$

and

$$
\begin{aligned}
\alpha_{n} \delta & <\alpha_{n} \Rightarrow \frac{\alpha_{n} \delta}{1-\left(1-\alpha_{n}\right) \delta\left[\beta_{n}+\delta\left(1-\beta_{n}\right)\right]} \\
& <\frac{\alpha_{n}}{1-\left(1-\alpha_{n}\right) \delta\left[\beta_{n}+\delta\left(1-\beta_{n}\right)\right]} \\
& \Rightarrow\left(\frac{\alpha_{n} \delta}{1-\left(1-\alpha_{n}\right) \delta\left[\beta_{n}+\delta\left(1-\beta_{n}\right)\right]}\right)^{n} \\
& <\left(\frac{\alpha_{n}}{1-\left(1-\alpha_{n}\right) \delta\left[\beta_{n}+\delta\left(1-\beta_{n}\right)\right]}\right)^{n} .
\end{aligned}
$$


Hence, we have

$$
\begin{aligned}
\lim _{n \rightarrow \infty} \frac{a_{n}}{c_{n}} & =\lim _{n \rightarrow \infty} \frac{\left(\frac{\alpha_{n} \delta}{1-\left(1-\alpha_{n}\right) \delta\left[\beta_{n}+\delta\left(1-\beta_{n}\right)\right]}\right)^{n} d\left(x_{0}, p\right)}{\left(\frac{\alpha_{n}}{1-\left(1-\alpha_{n}\right) \delta}\right)^{n} d\left(x_{0}, p\right)} \\
& =0,
\end{aligned}
$$

and

$$
\begin{aligned}
\lim _{n \rightarrow \infty} \frac{a_{n}}{b_{n}} & =\lim _{n \rightarrow \infty} \frac{\left(\frac{\alpha_{n} \delta}{1-\left(1-\alpha_{n}\right) \delta\left[\beta_{n}+\delta\left(1-\beta_{n}\right)\right]}\right)^{n} d\left(x_{0}, p\right)}{\left(\frac{\alpha_{n}}{1-\left(1-\alpha_{n}\right) \delta\left[\beta_{n}+\delta\left(1-\beta_{n}\right)\right]}\right)^{n} d\left(x_{0}, p\right)} \\
& =0 .
\end{aligned}
$$

We now support our above analytical proof by a numerical example using MATLAB.

Example 1. Let $E=[0,1]$ and $T: E \rightarrow E$ a mapping defined by $T x=\frac{x}{2}$. Note that $T$ is a contractive type mapping. Choose $\alpha_{n}=1-\frac{1}{n}$ and $\beta_{n}=1-\frac{1}{n}, n \geq 2$ and for $n=1$, $\alpha_{n}=\beta_{n}=0$. The comparison of the convergences of the implicit S-iteration (5), implicit Ishikawa type (6) and implicit Mann type iterations (7) to the fixed point $p=0$ are given in table with the initial value $x_{1}=1$.

The following table presents a comparison of rate of convergence of the implicit $S$ iteration process with implicit Ishikawa type and implicit Mann type iteration processes for the mapping given in Example 1.

\begin{tabular}{|c|c|c|c|}
\hline $\boldsymbol{n}$ & $\boldsymbol{I M I}$ & $\boldsymbol{I I I}$ & $\boldsymbol{I S I}$ \\
\hline 2 & 0.666666666666667 & 0.615384615384615 & 0.307692307692308 \\
\hline 5 & 0.406349206349206 & 0.352704628530670 & 0.022044039283167 \\
\hline 7 & 0.340992340992341 & 0.292145335107371 & 0.004564770861053 \\
\hline 10 & 0.283773192751521 & 0.240691952056443 & 0.000470101468860 \\
\hline 13 & 0.248169351176485 & 0.209336831746067 & 0.000051107624938 \\
\hline 16 & 0.223294138742407 & 0.187699995568689 & 0.000005728149279 \\
\hline 20 & 0.199408653447441 & 0.167113839554526 & 0.000000318744353 \\
\hline 25 & 0.178133771931084 & 0.148920204678483 & 0.000000008876336 \\
\hline 30 & 0.162477710197415 & 0.135609685643003 & 0.000000000252593 \\
\hline 35 & 0.150335628473559 & 0.125328510781087 & 0.000000000007295 \\
\hline 40 & 0.140563343828096 & 0.117078595772533 & 0.000000000000213 \\
\hline 43 & 0.135541774913220 & 0.112847389889567 & 0.000000000000026 \\
\hline 46 & 0.131022580805197 & 0.109043978938918 & 0.000000000000003 \\
\hline 50 & 0.125645129018549 & 0.104523598655989 & 0.000000000000000 \\
\hline
\end{tabular}

Remark 2. From the example above, we see that our iteration ISI (implicit S-iteration) is faster than the III (implicit Ishikawa iteration) and IMI (implicit Mann iteration) under the same control conditions. 
The following figure is a graphical presentation of the above results.
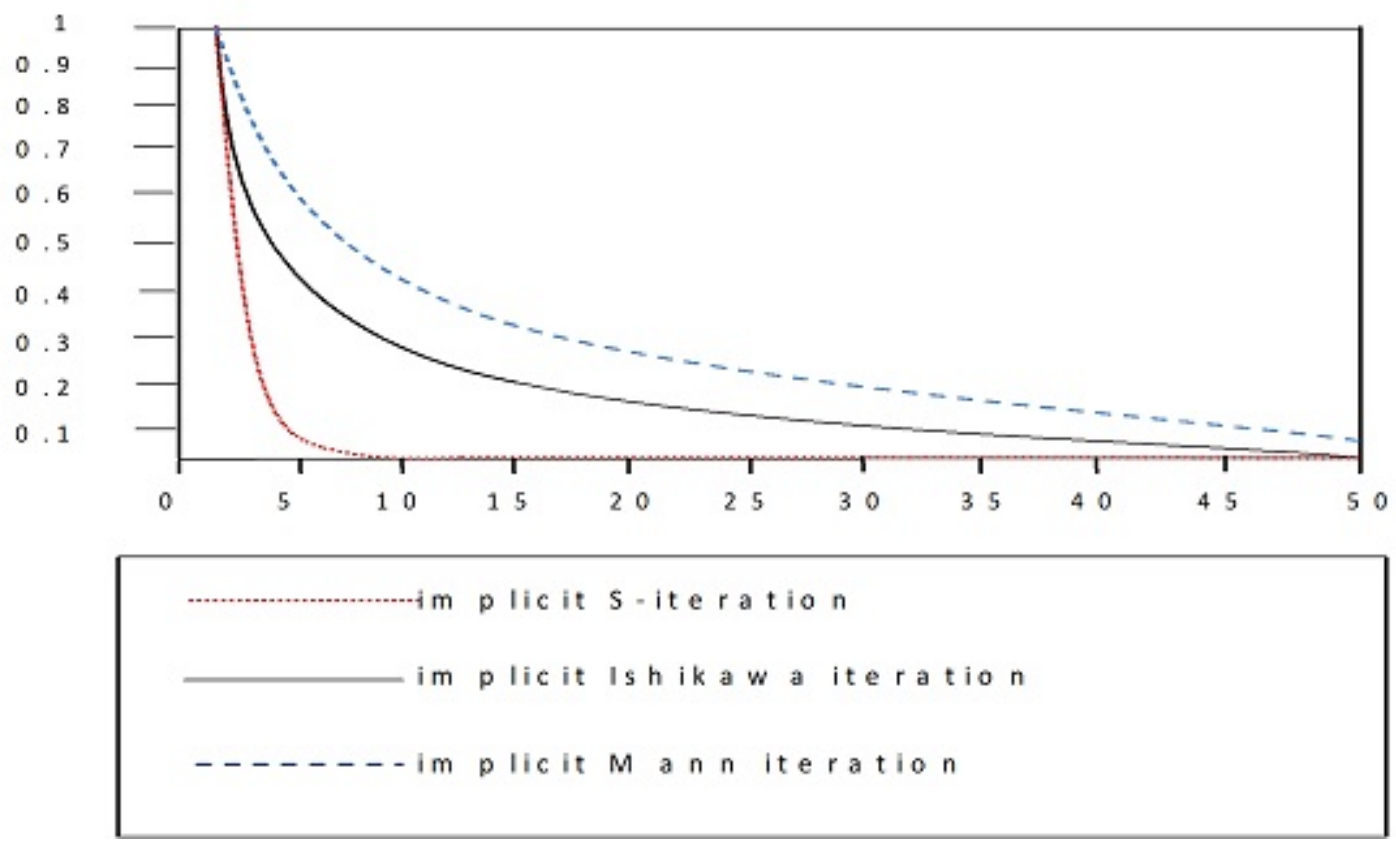

This figure confirm that the iteration (5) is converges faster than the iteration methods mentioned above.

Finally, we present a data dependence result for contractive type mapping $T$ using implicit S-iteration process.

Theorem 3. Let $E$ be a nonempty closed convex subset of $W$-hyperbolic space $X, T$ : $E \rightarrow E$ a contractive type mapping, $S$ an approximate operator of $T$ and $\left\{x_{n}\right\}$ a sequence defined in (5). For contractive type mapping $T$, define an iterative sequence $\left\{u_{n}\right\}$ as follows:

$$
\begin{aligned}
& u_{n}=W\left(S u_{n-1}, T v_{n}, \alpha_{n}\right) \\
& v_{n}=W\left(u_{n}, S u_{n}, \beta_{n}\right), \quad n \in \mathbb{N},
\end{aligned}
$$

where $\left\{a_{n}\right\}$ and $\left\{\beta_{n}\right\}$ are real sequences in $[0,1]$ satisfying $a_{n}<1, n \in \mathbb{N}$, and $\sum\left(1-\alpha_{n}\right)=$ $\infty$. If $T p=p$ and $S q=q$ such that $u_{n} \rightarrow q$ as $n \rightarrow \infty$, then we have, $d(p, q) \leq \frac{2 \varepsilon}{(1-\delta)^{2}}$, $\varepsilon>0$ is some appropriate number.

Proof. By (4), (5) and (24), we have

$$
\begin{aligned}
d\left(y_{n}, v_{n}\right) & =d\left(W\left(x_{n}, T x_{n}, \beta_{n}\right), W\left(u_{n}, S u_{n}, \beta_{n}\right)\right) \\
& \leq \beta_{n} d\left(x_{n}, u_{n}\right)+\left(1-\beta_{n}\right) d\left(T x_{n}, S u_{n}\right) \\
& \leq \beta_{n} d\left(x_{n}, u_{n}\right)+\left(1-\beta_{n}\right)\left[d\left(T x_{n}, S u_{n}\right)+d\left(T u_{n}, S u_{n}\right)\right] \\
& \leq \beta_{n} d\left(x_{n}, u_{n}\right)+\left(1-\beta_{n}\right)\left[\delta d\left(x_{n}, u_{n}\right)+\varphi\left(d\left(x_{n}, T x_{n}\right)\right)+\varepsilon\right]
\end{aligned}
$$


I. Yildirim, M. Abbas / Eur. J. Pure Appl. Math, 11 (1) (2018), 189-201

$$
=\left[\beta_{n}+\left(1-\beta_{n}\right) \delta\right] d\left(x_{n}, u_{n}\right)+\left(1-\beta_{n}\right)\left[\varphi\left(d\left(x_{n}, T x_{n}\right)\right)+\varepsilon\right]
$$

and

$$
\begin{aligned}
d\left(x_{n}, u_{n}\right)= & d\left(W\left(T x_{n-1}, T y_{n}, \alpha_{n}\right), W\left(S u_{n-1}, S v_{n}, \alpha_{n}\right)\right) \\
\leq & \alpha_{n} d\left(T x_{n-1}, S u_{n-1}\right)+\left(1-\alpha_{n}\right) d\left(T y_{n}, S v_{n}\right) \\
\leq & \alpha_{n}\left[d\left(T x_{n-1}, T u_{n-1}\right)+d\left(T u_{n-1}, S u_{n-1}\right)\right] \\
& +\left(1-\alpha_{n}\right)\left[d\left(T y_{n}, T v_{n}\right)+d\left(T v_{n}, S v_{n}\right)\right] \\
\leq & \alpha_{n}\left[\delta d\left(x_{n-1}, u_{n-1}\right)+\varphi\left(d\left(x_{n-1}, T x_{n-1}\right)\right)+\varepsilon\right] \\
& +\left(1-\alpha_{n}\right)\left[\delta d\left(y_{n}, v_{n}\right)+\varphi\left(d\left(y_{n}, T y_{n}\right)\right)+\varepsilon\right] \\
= & \alpha_{n} \delta d\left(x_{n-1}, u_{n-1}\right)+\alpha_{n} \varphi\left(d\left(x_{n-1}, T x_{n-1}\right)\right)+\varepsilon \\
& +\left(1-\alpha_{n}\right) \delta d\left(y_{n}, v_{n}\right)+\left(1-\alpha_{n}\right) \varphi\left(d\left(y_{n}, T y_{n}\right)\right) .
\end{aligned}
$$

Substituting (25) in (26), we obtain that

$$
\begin{aligned}
d\left(x_{n}, u_{n}\right) \leq & \alpha_{n} \delta d\left(x_{n-1}, u_{n-1}\right)+\alpha_{n} \varphi\left(d\left(x_{n-1}, T x_{n-1}\right)\right) \\
& +\left(1-\alpha_{n}\right) \varphi\left(d\left(y_{n}, T y_{n}\right)\right)+\varepsilon+\left(1-\alpha_{n}\right) \delta \\
& {\left[\left[\beta_{n}+\left(1-\beta_{n}\right) \delta\right] d\left(x_{n}, u_{n}\right)+\left(1-\beta_{n}\right)\left[\varphi\left(d\left(x_{n}, T x_{n}\right)\right)+\varepsilon\right]\right] } \\
= & \alpha_{n} \delta d\left(x_{n-1}, u_{n-1}\right)+\alpha_{n} \varphi\left(d\left(x_{n-1}, T x_{n-1}\right)\right) \\
& +\left(1-\alpha_{n}\right) \varphi\left(d\left(y_{n}, T y_{n}\right)\right)+\varepsilon+\left(1-\alpha_{n}\right) \delta \\
& {\left[\beta_{n}+\left(1-\beta_{n}\right) \delta\right] d\left(x_{n}, u_{n}\right)+\left(1-\alpha_{n}\right) \delta } \\
& \left(1-\beta_{n}\right) \varphi\left(d\left(x_{n}, T x_{n}\right)\right)+\left(1-\alpha_{n}\right) \delta\left(1-\beta_{n}\right) \varepsilon .
\end{aligned}
$$

It follows from ( 27) that

$$
\begin{aligned}
& {\left[1-\left(1-\alpha_{n}\right) \delta\left[\beta_{n}+\left(1-\beta_{n}\right) \delta\right]\right] d\left(x_{n}, u_{n}\right) } \\
& \leq \quad \alpha_{n} \delta d\left(x_{n-1}, u_{n-1}\right)+\alpha_{n} \varphi\left(d\left(x_{n-1}, T x_{n-1}\right)\right) \\
&+\left(1-\alpha_{n}\right) \varphi\left(d\left(y_{n}, T y_{n}\right)\right)+\varepsilon+\left(1-\alpha_{n}\right) \delta \\
& \\
&\left(1-\beta_{n}\right) \varphi\left(d\left(x_{n}, T x_{n}\right)\right)+\left(1-\alpha_{n}\right) \delta\left(1-\beta_{n}\right) \varepsilon
\end{aligned}
$$

which further implies that

$$
\begin{aligned}
& d\left(x_{n}, u_{n}\right) \leq \frac{\alpha_{n} \delta}{1-\left(1-\alpha_{n}\right) \delta\left[\beta_{n}+\left(1-\beta_{n}\right) \delta\right]} d\left(x_{n-1}, u_{n-1}\right) \\
& +\frac{\left\{\begin{array}{c}
\alpha_{n} \varphi\left(d\left(x_{n-1}, T x_{n-1}\right)\right)+\left(1-\alpha_{n}\right) \varphi\left(d\left(y_{n}, T y_{n}\right)\right) \\
+\left(1-\alpha_{n}\right) \delta\left(1-\beta_{n}\right) \varphi\left(d\left(x_{n}, T x_{n}\right)\right)
\end{array}\right\}}{1-\left(1-\alpha_{n}\right) \delta\left[\beta_{n}+\left(1-\beta_{n}\right) \delta\right]} \\
& \frac{+\varepsilon+\left(1-\alpha_{n}\right) \delta\left(1-\beta_{n}\right) \varepsilon}{1-\left(1-\alpha_{n}\right) \delta\left[\beta_{n}+\left(1-\beta_{n}\right) \delta\right]} .
\end{aligned}
$$

We set

$$
\Delta_{n}=\frac{\alpha_{n} \delta}{1-\left(1-\alpha_{n}\right) \delta\left[\beta_{n}+\left(1-\beta_{n}\right) \delta\right]} .
$$


Following arguments similar to those in the proof of Theorem 1, we have

$$
\Delta_{n} \leq 1-\left(1-\alpha_{n}\right)(1-\delta)
$$

Therefore,

$$
\begin{aligned}
d\left(x_{n}, u_{n}\right) \leq & {\left[1-\left(1-\alpha_{n}\right)(1-\delta)\right] d\left(x_{n-1}, u_{n-1}\right) } \\
+ & \begin{array}{c}
\left(1-\alpha_{n}\right)(1-\delta)\left\{\begin{array}{c}
\frac{\alpha_{n}}{1-\alpha_{n}} \varphi\left(d\left(x_{n-1}, T x_{n-1}\right)\right)+\varphi\left(d\left(y_{n}, T y_{n}\right)\right) \\
+\delta\left(1-\beta_{n}\right) \varphi\left(d\left(x_{n}, T x_{n}\right)\right)+2 \varepsilon
\end{array}\right\} \\
(1-\delta)\left[1-\left(1-\alpha_{n}\right) \delta\left[\beta_{n}+\left(1-\beta_{n}\right) \delta\right]\right]
\end{array}
\end{aligned}
$$

Note that

$$
\begin{aligned}
1-\left(1-\alpha_{n}\right) \delta\left[\beta_{n}+\left(1-\beta_{n}\right) \delta\right] & =1-\left(1-\alpha_{n}\right) \delta\left[1-\left(1-\beta_{n}\right)(1-\delta)\right] \\
& \geq 1-\delta
\end{aligned}
$$

Hence

$$
\begin{aligned}
d\left(x_{n}, u_{n}\right) \leq & {\left[1-\left(1-\alpha_{n}\right)(1-\delta)\right] d\left(x_{n-1}, u_{n-1}\right) } \\
+ & \begin{array}{c}
\left(1-\alpha_{n}\right)(1-\delta)\left\{\begin{array}{c}
\frac{\alpha_{n}}{1-\alpha_{n}} \varphi\left(d\left(x_{n-1}, T x_{n-1}\right)\right)+\varphi\left(d\left(y_{n}, T y_{n}\right)\right) \\
+\delta\left(1-\beta_{n}\right) \varphi\left(d\left(x_{n}, T x_{n}\right)\right)+2 \varepsilon
\end{array}\right\} \\
(1-\delta)^{2}
\end{array}
\end{aligned}
$$

That is,

$$
a_{n+1} \leq\left(1-\mu_{n}\right) a_{n}+\mu_{n} \eta_{n}
$$

where $a_{n+1}=d\left(x_{n}, u_{n}\right), \mu_{n}=\left(1-\alpha_{n}\right)(1-\delta)$ and

$$
\eta_{n}=\frac{\begin{array}{c}
\frac{\alpha_{n}}{1-\alpha_{n}} \varphi\left(d\left(x_{n-1}, T x_{n-1}\right)\right)+\varphi\left(d\left(y_{n}, T y_{n}\right)\right) \\
+\delta\left(1-\beta_{n}\right) \varphi\left(d\left(x_{n}, T x_{n}\right)\right)+2 \varepsilon
\end{array}}{(1-\delta)^{2}} .
$$

From Theorem 1, we have $\lim _{n \rightarrow \infty} d\left(x_{n}, p\right)=\lim _{n \rightarrow \infty} d\left(x_{n-1}, p\right)=0$, and $\lim _{n \rightarrow \infty} d\left(u_{n}, p\right)=$ 0 . As $\varphi$ is continuous, $\lim _{n \rightarrow \infty} \varphi\left(d\left(x_{n-1}, T x_{n-1}\right)\right)=0, \lim _{n \rightarrow \infty} \varphi\left(d\left(y_{n}, T y_{n}\right)\right)=0$, and $\lim _{n \rightarrow \infty} \varphi\left(d\left(x_{n}, T x_{n}\right)\right)=0$. Thus all the conditions of Lemma 1 are satisfied, therefore (31) becomes

$$
d(p, q) \leq \frac{2 \varepsilon}{(1-\delta)^{2}}
$$

\section{References}

[1] M. Abbas, P. Vetro, S. H. Khan. On fixed points of Berinde's contractive mappings in cone metric spaces. Carpath. J. Math., 26(2):121-133, 2010. 
[2] S. Banach. Sur les opérations dans les ensembles abstraits et leur applications aux équations intégrales. Fund. Math., 3:133-181, 1922.

[3] V. Berinde. Picard iteration converges faster than Mann iteration for a class of quasicontractive operators, Fixed Point Theory and Applications, 2004:97-105, 2004.

[4] V. Berinde. On the convergence of the Ishikawa iteration in the class of quasi contractive operators. Acta Math. Univ. Comen. 73:119-126, 2004.

[5] S.S. Chang, L. Yang, X.R. Wang. Stronger convergence theorems for an infinite family of uniformly quasi-Lipschitzian mappings in convex metric spaces. Appl. Math. Comp. 217:277-282, 2010 .

[6] R. Chugh, P. Malik and V. Kumar. On analytical and numerical study of implicit fixed point iterations. Cogent Mathematics, 2:1021623, 2015.

[7] Lj. B. Ćirić, Rafiq, A., Cakić, N., \& Ume, J. S. Implicit Mann fixed point iterations for pseudo-contractive mappings. Applied Mathematics Letters, 22:581-584, 2009.

[8] Lj. B. Ćirić, Rafiq, A., Radenović, S., Rajović, M., \& Ume, J. S. On Mann implicit iterations for strongly accretive and strongly pseudo-contractive mappings. Applied Mathematics and Computation, 198:128-137, 2008.

[9] Lj. B.Ciric, Ume, J. S. M., \& Khan, S. On the convergence of the Ishikawa iterates to a common fixed point of two mappings. Archivum Mathematicum (Brno) Tomus, 39:123-127, 2003.

[10] C.O. Imoru, M.O. Olantiwo. On the stability of Picard and Mann iteration processes. Carpath. J. Math. 19:155-160, 2003.

[11] A.R. Khan, M.A. Ahmed. Convergence of a general iterative scheme for a finite family of asymptotically quasi-nonexpansive mappings in convex metric spaces and applications. Comput. Math. Appl. 59:2990-2995, 2015.

[12] S.H. Khan, I. Yildirim, M. Ozdemir. Convergence of an implicit algorithm for two families of nonexpansive mappings. Comput. Math. Appl. 59:3084-3091, 2010.

[13] S. H. Khan. Common fixed points of quasi-contractive type operators by a generalized iterative process. IAENG Int. J. Appl. Math., 41(3):260-264, 2011.

[14] J. K. Kim, K. S. Kim, S. M. Kim. Convergence theorems of implicit iteration process for for finite family of asymptotically quasi-nonexpansive mappings in convex metric space. Nonlinear Analysis and Convex Analysis, 1484:40-51, 2006.

[15] U. Kohlenbach. Some logical metatherems with applications in functional analysis. Transactions of the American Mathematical Society, 357:89-128, 2004. 
[16] Q.Y. Liu, Z.B. Liu, N.J. Huang. Approximating the common fixed points of two sequences of uniformly quasi-Lipschitzian mappings in convex metric spaces. Appl. Math. Comp. 216:883-889, 2010.

[17] M.O. Osilike, A. Udomene. Short proofs of stability results for fixed point iteration procedures for a class of contractive-type mappings. Indian J. Pure Appl. Math. 30:1229-1234, 1999.

[18] S.M. Şoltuz, T. Grosan. Data dependence for Ishikawa iteration when dealing with contractive like operators. Fixed Point Theory Appl. Article ID 242916 (2008). doi:10.1155/2008/242916, 2008.

[19] W. Takahashi. A convexity in metric space and nonexpansive mappings. Kodai Math. Sem. Rep. 22:142-149, 1970.

[20] I. Yildirim, S. H. Khan. Convergence theorems for common fixed points of asymptotically quasi-nonexpansive mappings in convex metric spaces. Applied Mathematics and Computation, 218(9):4860-4866, 2012.

[21] T. Zamfirescu. Fix point theorems in metric spaces. Arch. Math. 23:292-298, 1972. 